\title{
UCLA
}

\section{Earthquake Engineering}

\section{Title}

Finite Element Modeling of Shallow Foundations on Nonlinear Soil Medium

Permalink

https://escholarship.org/uc/item/8b43q93s

\section{Authors}

Zhang, Jian

Tang, Yuchuan

Publication Date

2007 


\title{
Finite Element Modeling of Shallow Foundations on Nonlinear Soil Medium
}

\author{
Authors: \\ Jian Zhang, University of California, Los Angeles, zhangj@ucla.edu \\ Yuchuan Tang, University of California, Los Angeles, bytang@ucla.edu
}

\begin{abstract}
This paper investigates the dynamic response of shallow foundations on linear and nonlinear soil medium using finite element method. The study was motivated by the need to develop macroscopic foundation models that can realistically capture the nonlinear behavior and energy dissipation mechanism of shallow foundations. An infinitely long strip foundation resting on soil half-space is analyzed in depth to evaluate the dependence of its dynamic responses on various parameters, e.g. foundation width, material properties, input motion amplitude and frequency etc. Special attentions are paid to choose appropriate domain scale, mesh size and boundary conditions so as to minimize the often observed numerical oscillations when the outgoing waves are contaminated by the reflecting waves at boundaries. Such judicious choice results in an excellent agreement between the finite element analysis and the analytical solution of strip foundation on linear soil half-space. Closed-form formulas are developed to describe the frequency-dependent linear dynamic stiffness of strip foundation along both horizontal and vertical directions. Various nonlinear constitutive models of soil, which exhibit the yielding and kinematic hardening behavior of soil, are implemented in this study to evaluate the dynamic stiffness of strip foundation sitting on nonlinear soil medium. The finite element analyses reveal the strong dependency of response on input motion amplitude, frequency and yielding of soil. A nonlinearity indicator is developed to incorporate the combined effects of initial elastic stiffness, yielding stress and excitation amplitude. The numerical analyses presented here provide improved understanding on the nonlinear behavior and energy dissipation mechanism of shallow foundations under dynamic loads.
\end{abstract}

\section{INTRODUCTION}

It is recognized that the dynamic responses of structures with flexible foundations are affected by the nonlinear dynamic behavior of individual components as well as the interaction between them, i.e. the soil-structure interaction effects. These interaction effects are often characterized by changing stiffness and energy dissipation through either hysteretic or radiation damping [1]. They can be represented by frequency dependent dynamic stiffness, which subsequently provides information on equivalent spring and dashpot constants of foundations. 
Various analytical models are available to describe the dynamic stiffness of shallow foundations of different shapes on elastic soil medium, e.g. strip foundation on elastic half-space [2,3] and on visco-elastic soil layer [4,5], circular foundation on elastic halfspace [6,7] and on visco-elastic half-space [8], rectangular foundation on elastic halfspace or layered medium $[9,10]$, and cylindrical and rectangular embedded foundations [11]. Gazetas [12] and Mylonakis et al. [13] compiled an extensive set of graphs and tables for dynamic stiffness of foundations with a variety of geometries and linear soil conditions. Despite the abundance of analytical solutions for shallow foundations on linear soil medium, very limited work has been reported on the dynamic stiffness of shallow foundations on nonlinear soil medium [13]. The nonlinearity of soil has caused reduced stiffness and modified energy dissipation mechanism. As pointed out by Borja and his co-workers [14,15], the local yielding in an otherwise homogeneous elastic soil half-space tends to reduce the radiation damping and create resonance frequencies.

In this study, finite element method is adopted to compute the dynamic response of an infinitely long strip foundation resting on an elastic and inelastic half-space. Numerical results from finite element method are compared with the theoretical solution of strip foundation on elastic half-space so as to provide guidance on choosing appropriate domain scale, mesh size and boundary condition for correct modeling of the wave propagation in a half-space. Closed-form formulas are developed to describe the frequency-dependent linear dynamic stiffness of strip foundation along both horizontal and vertical directions. Subsequently, the strip foundation on nonlinear soil medium is analyzed. Nonlinear soil models that exhibit yielding and kinematic hardening are implemented based on a simple procedure derived from widely available shear modulus reduction curves. The dynamic stiffness is evaluated and effects of foundation width, input motion amplitude and frequency, and development of soil nonlinearity are quantified by a newly developed nonlinearity indicator. The numerical results showed here revealed that the energy dissipation through radiation damping of nonlinear soil is significantly reduced due to localized yielding zone in soil.

\section{DyNAMIC STIFFNESS OF STRIP FOUNDATION ON ELASTIC SOIL}

Consider an infinitely-long rigid strip foundation sitting on elastic soil half-space subject to harmonic excitations, as shown in Figure 1. Its dynamic stiffness can be obtained analytically $[2,3]$. Under a harmonic motion, the reacting forces are related to displacements by the general form shown below:

$$
\left\{\begin{array}{l}
P_{v}(t) \\
P_{h}(t)
\end{array}\right\}=\pi G\left[\begin{array}{cc}
c_{11}+\mathrm{i} d_{11} & 0 \\
0 & c_{22}+\mathrm{i} d_{22}
\end{array}\right]\left\{\begin{array}{l}
U_{v}(t) \\
U_{h}(t)
\end{array}\right\}
$$

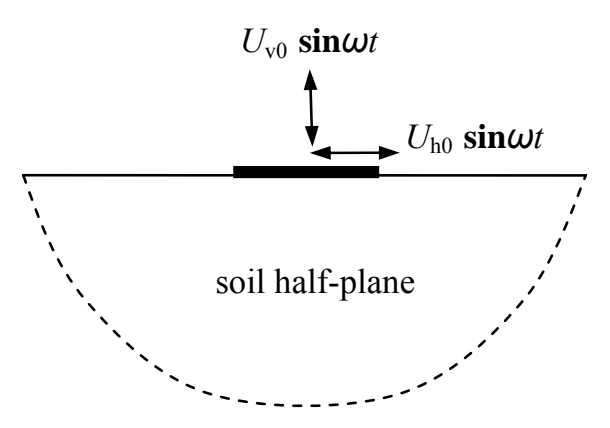

FIGURE 1

FOUNDATION GEOMETRY AND EXCITATION CONDITIONS

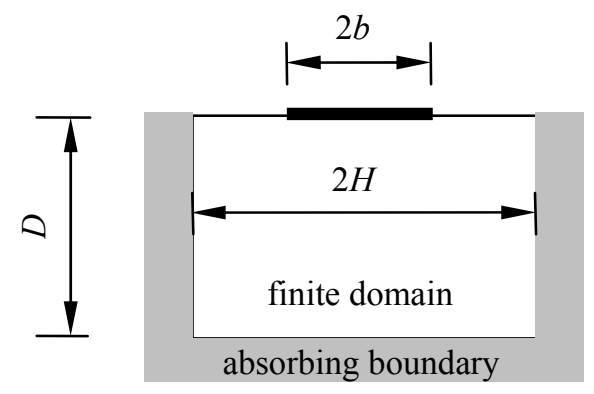

FIGURE 2

FINITE DOMAIN AND ABSORBING BOUNDARY 
where $G$ is the shear modulus of soil, $\pi G\left(c_{11}+\mathrm{i} d_{11}\right)$ and $\pi G\left(c_{22}+\mathrm{i} d_{22}\right)$ are the dynamic stiffnesses in vertical and horizontal directions respectively. The force-displacement relationship in (1) is analogous to that of a spring-dashpot system with spring constant $\pi G c_{11}$ (or $\pi G c_{22}$ ) and dashpot coefficient $\pi G d_{11} / \omega$ (or $\pi G d_{22} / \omega$ ). The dynamic stiffness parameters $c_{11}, d_{11}, c_{22}, d_{22}$ depend on both the frequency of excitation and soil properties and are often plotted against dimensionless frequency $a_{0}=\omega b / v_{\mathrm{s}}$ for a given Poisson's ratio with $b$ as the half-width of strip foundation and $v_{\mathrm{s}}$ as the shear wave velocity of soil.

Finite element method is used in this study to conduct the dynamic analyses of strip foundation under harmonic displacement excitation in vertical and horizontal directions respectively. The soil half-space is represented by a finite domain where an absorbing boundary condition needs to be present to correctly model the outgoing waves of an infinite medium (Figure 2). Maximum element size, boundary conditions and scale of the finite domain dominate the accuracy of finite element analysis. Previous research [16,17] suggested that the maximum element size $l_{\max }$ should satisfy

$$
l_{\max } \leq\left(\frac{1}{8} \sim \frac{1}{5}\right) L
$$

where $L$ is shear wave length. For a given finite element mesh, (2) equivalently puts an upper limit on the applicable dimensionless excitation frequency $a_{0}$ :

$$
a_{0} \leq\left(\frac{1}{8} \sim \frac{1}{5}\right) \frac{2 \pi b}{l_{\max }}
$$

An absorbing boundary is also required to simulate the waves transmitting outward in a half-space. Either viscous damping boundary [18] or infinite element boundary [19] can be used for this purpose. However, their implementation in ABAQUS results in unexpected numerical oscillations as observed in Figure 3, where the dynamic stiffness parameters $c_{11}$ and $c_{22}$ are plotted for a finite domain of $D=H=10 b$ with absorbing boundaries. Similar oscillation has been observed for a even larger domain of $D=H=20 b$.

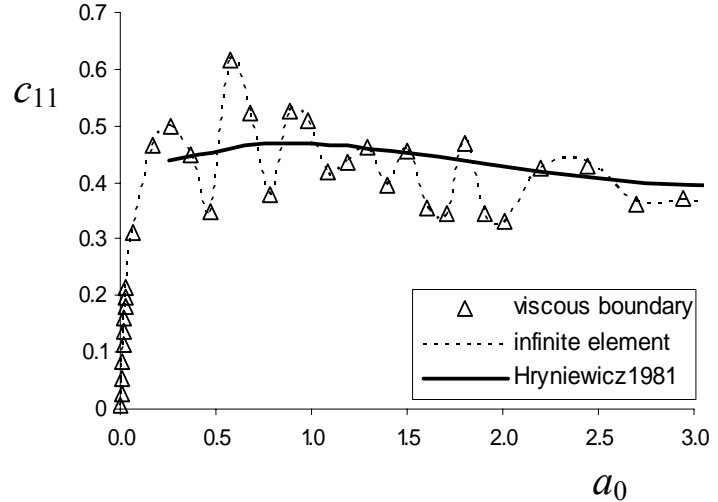

(a)

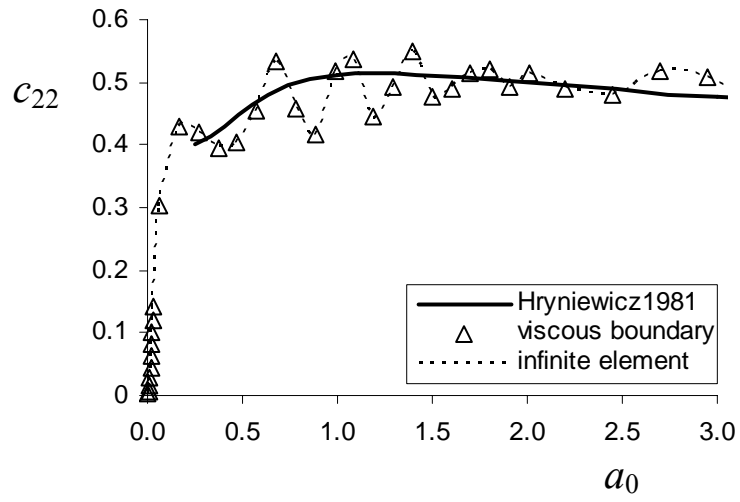

(b)

FIGURE 3

DYNAMIC STIFFNESS OF STRIP FOUNDATION COMPUTED BY FEM (WITH NUMERICAL OSCILLATION) AND ANALYTICAL SOlUtion For Horizontal (a) AND VERTICAL (b) DirECTIONS

To eliminate the oscillation, a large finite domain is needed so that the steady state response can be achieved before the wave reflection at boundary contaminates the response [14]. For this purpose, the scale of the finite domain needs to satisfy 


$$
n T v_{p} \leq L_{r}
$$

where $L_{\mathrm{r}}$ is the length of the shortest wave reflection path within the finite domain, $v_{\mathrm{p}}$ is the longitudinal wave velocity, $T$ is the period of harmonic excitation, $n$ is the number of periods from beginning of excitation which includes one full cycle of steady state response. Substituting dimensionless frequency $a_{0}$ into (4) for period $T$ yields the lower bound on the applicable dimensionless excitation frequency

$$
2 n \pi \frac{b}{L_{r}} \cdot \sqrt{\frac{2-2 v}{1-2 v}} \leq a_{0}
$$

A finite mesh of $H=250 \mathrm{~m}, D=250 \mathrm{~m}, l_{\max }=1.25 \mathrm{~m}$ (refer to Figure 2) was set up for a strip foundation of half-width $b=1 \mathrm{~m}$ on elastic soil medium of $v_{\mathrm{s}}=201.5 \mathrm{~m} / \mathrm{s}, v=0.25$, $\rho=1600 \mathrm{~kg} / \mathrm{m}^{3}$. Equation (5) gives lower bound of excitation frequency as $a_{0} \geq 0.04$ while (3) gives upper bound of excitation frequency as $a_{0} \leq 1.0$. For input frequency within this range, the numerical oscillation is well eliminated. Figure 4 compares the dynamic stiffness parameters computed by finite element and the theoretical solution given by Hryniewicz [3], where an excellent agreement is achieved.

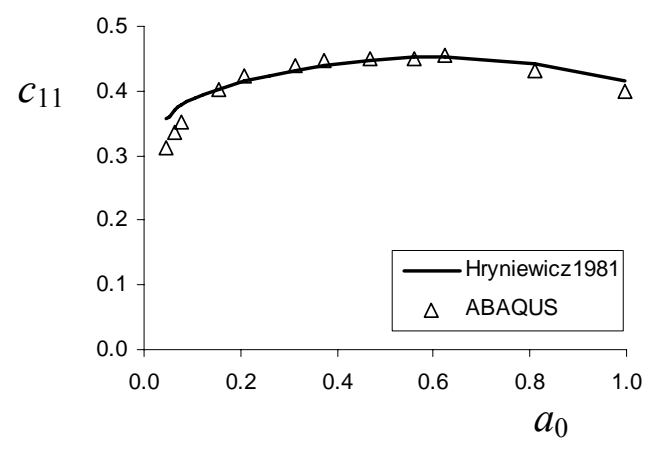

(a)

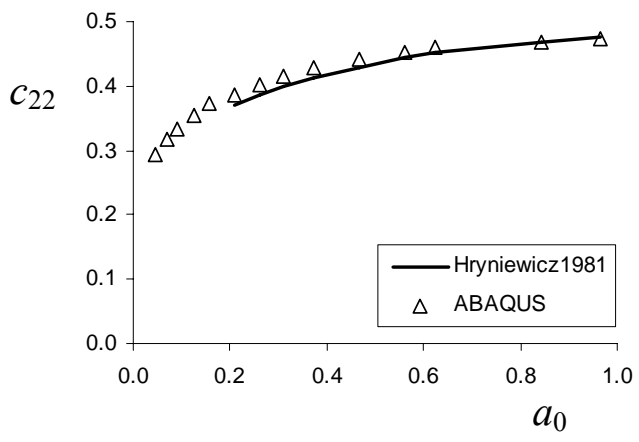

(c)

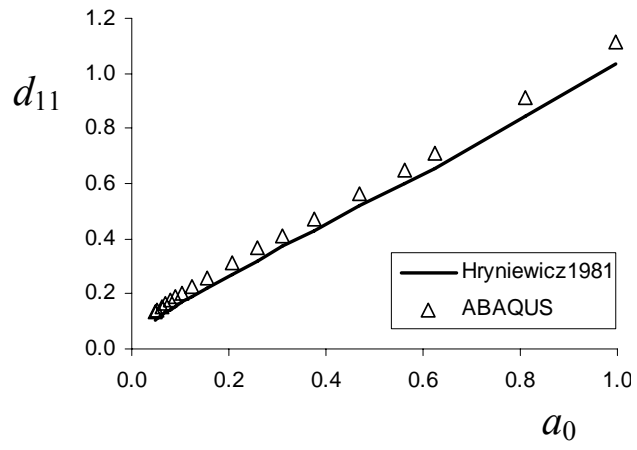

(b)

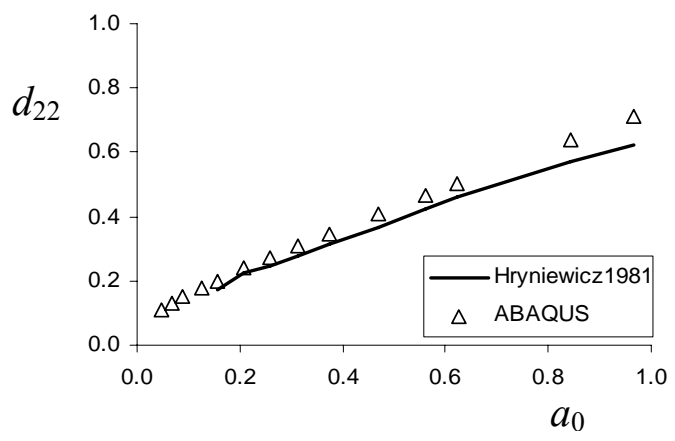

(d)

FIGURE 4

DYNAMIC STIFFNESS OF STRIP FoundATION FOR VERTICAL $(a, b)$ AND HORIZONTAL DiRECTIONS (c,d)

The dynamic stiffness of strip foundation depends on foundation width, Young's modulus and Poissson's ratio of soil. The first two parameters can be incorporated by using dimensionless frequency $a_{0}$. Simplified formulas are developed to characterize the effects of Poisson's ratio as shown in (6) to (9). These simplified formulas showed excellent agreement with finite element results as shown in Figure 5, where the family of 
curves corresponding to different Poisson's ratio are plotted for vertical (Figures 5a and $5 \mathrm{~b}$ ) and horizontal (Figures 5c and 5d) directions.

$$
\begin{gathered}
c_{11}=\frac{a_{0}}{1.2 a_{0}^{3}-1.1 a_{0}^{2}+2.4 a_{0}+0.04}+0.55(v-0.25) \\
d_{11}=\left(3.4 v^{2}-0.5 v+0.9\right) a_{0}+0.1 \\
c_{22}=\frac{a_{0}}{0.45 a_{0}^{3}-0.83 a_{0}^{2}+2.46 a_{0}+0.06}+[0.67(v-0.25)+0.01] \cdot\left(0.47 a_{0}+0.56\right) \\
d_{22}=0.65 a_{0}+0.1
\end{gathered}
$$

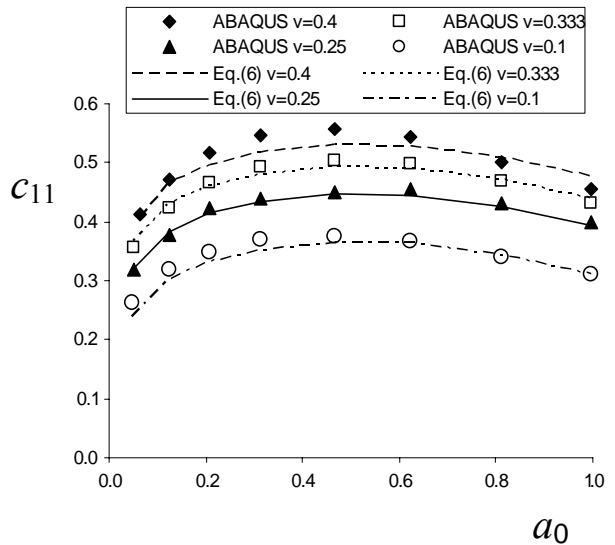

(a)

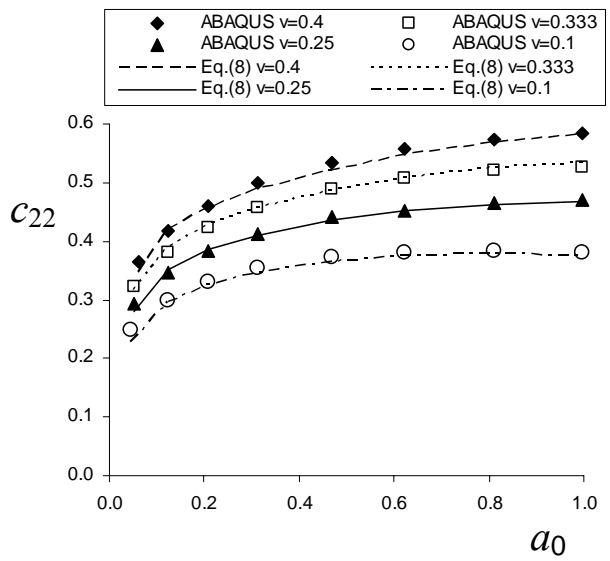

(c)

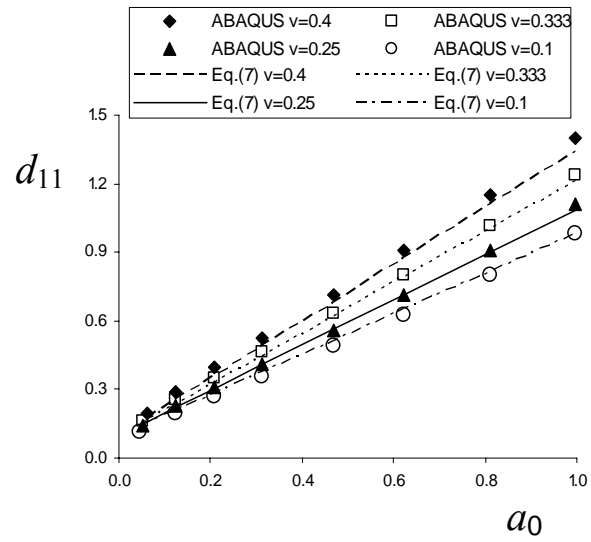

(b)

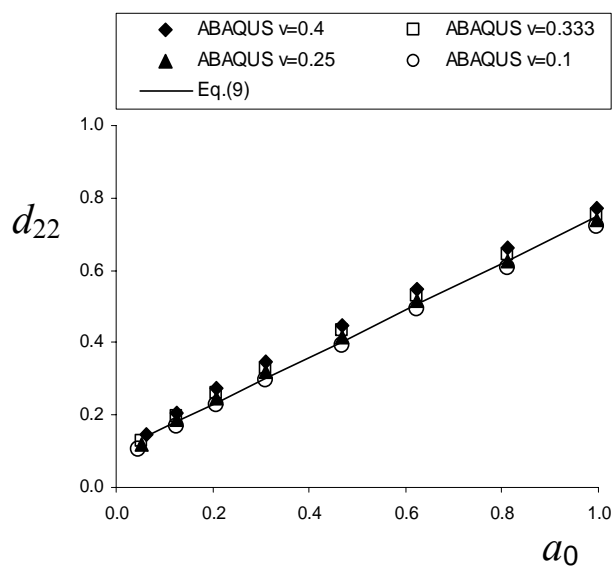

(d)

FIGURE 5

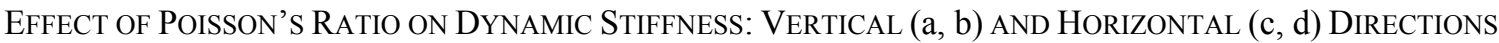

\section{RADIATION DAMPING OF STRIP FOUNDATION ON NONLINEAR SOIL}

During strong earthquakes, soil often behaves nonlinearly. The plasticity experienced in soil reduces the energy dissipated through outgoing waves. As result, radiation damping of nonlinear soil is quite different from that of linear soil. Finite element method is the only effective way to reveal the amplitude and frequency dependent nature of the foundation-nonlinear soil system. 
It is recognized that an infinitely long strip foundation on nonlinear soil medium behaves differently under static cyclic loading than under dynamic harmonic excitation, as shown in Figure 6. The area within static loop accounts for hysteretic energy $W_{\mathrm{h}}$, which is frequency independent. The equivalent hysteretic damping ratio of the nonlinear soil-foundation system can be defined as

$$
\xi=\frac{W_{h}}{4 \pi W_{s}} \times 100 \%
$$

where $W_{\mathrm{s}}$ represents the linear strain energy. On the other hand, the area within dynamic loop accounts for total dissipated energy $W_{\mathrm{d}}$ through both hysteretic and radiation damping, which depends on excitation frequency. The nonlinear radiation energy, $W_{\mathrm{r}}$, can be expressed as the difference between total dissipated energy and hysteretic energy as $W_{r}=W_{d}-W_{h}$. For elastic soil half-space, $W_{\mathrm{h}}=0$ and $W_{\mathrm{d}}=W_{\mathrm{r}}$.

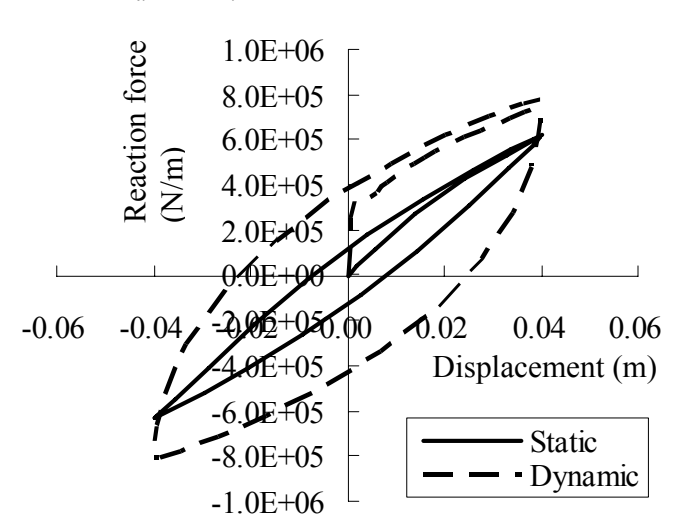

FIGURE 6

STATIC AND DYNAMIC RESPONSE OF NONLINEAR SOIL

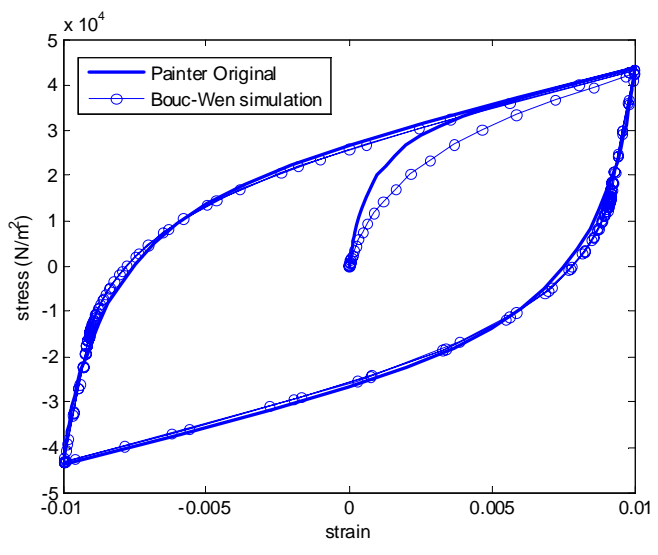

FIGURE 7

BOUC-WEN MODEL FOR SIMPLE SHEAR

A simple procedure has been developed to generate cyclic shear behavior of different soil types. The stress-strain backbone curve for simple shear can be easily obtained from widely available shear modulus reduction curves. A cyclic loop (thick curve in Figure 7) can be obtained by applying the Masing rule [20] to the backbone curve using the soil type data of Painter Street Bridge [21]. It is compared with the cyclic loop generated by Bouc-Wen model [22] (line with circles in Figure 7). Excellent agreement can be achieved for both sand and clay samples with a set of model parameters of Bouc-Wen model after trial-and-error adjusting. Following this procedure, four material models (Cases A, B, C and D) were generated (Figure 8). Case A differs from Case B in yielding stress $\tau_{\mathrm{y}}\left(3.0 \times 10^{4} \mathrm{~N} / \mathrm{m}^{2}\right.$ and $2.1 \times 10^{4} \mathrm{~N} / \mathrm{m}^{2}$ respectively) while it differs from Case $\mathrm{C}$ in post-yielding stiffness $\left(3.0 \times 10^{6} \mathrm{~N} / \mathrm{m}^{2}\right.$ and $4.2 \times 10^{6} \mathrm{~N} / \mathrm{m}^{2}$ respectively). Cases $A$ and D have different shear modulus $\mathrm{G}\left(6.0 \times 10^{7} \mathrm{~N} / \mathrm{m}^{2}\right.$ and $1.0 \times 10^{8} \mathrm{~N} / \mathrm{m}^{2}$ respectively $)$. Poisson's ratio of 0.25 is specified for all the four soil material cases.

An elasto-plastic constitutive model of von Mises yield criterion and nonlinear kinematic hardening rule in ABAQUS was selected to simulate nonlinear soil material. The model is defined by a few representative points on the steady-state cyclic loop given by Bouc-Wen model. As shown in Figure 9, the implemented model in ABAQUS is able to reproduce the intended cyclic behavior of soil.

Besides (3) and (5), the possible development of plasticity in soil medium should also be taken into account when set up finite element mesh for nonlinear dynamic analysis. 
Development of plasticity results in smaller wave velocity, which requires finer mesh in the yield zone. Two plane strain finite element meshes were set up for different excitation frequency ranges in order to satisfy all the requirements. Numerical results have shown that the soil density has minimum effect on dynamic stiffness (see Figure 10) while the amplitude of displacement excitation affects the development of nonlinearity in soil hence the nonlinear radiation damping. Figure 11 plots the nonlinear radiation damping for soil material Case A with density of $\rho=1600 \mathrm{~kg} / \mathrm{m}^{3}$ and different combinations of foundation half-width $b$ and displacement amplitude $U_{0}$. Larger $U_{0} / b$ results in more nonlinearity in soil, which leads to smaller radiation damping due to outgoing waves. Analyses with soil material Cases B, C and D reveal the similar trend.

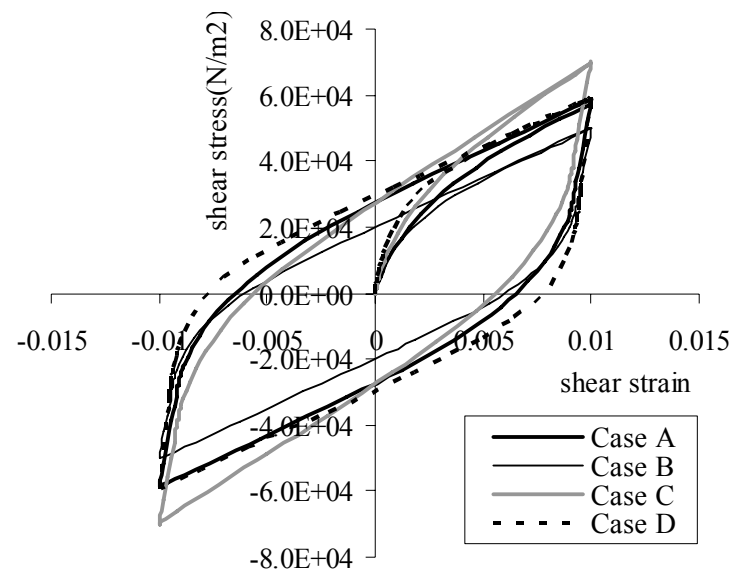

FIGURE 8

Four DisTinctive SoIL MATERIAL MODELS

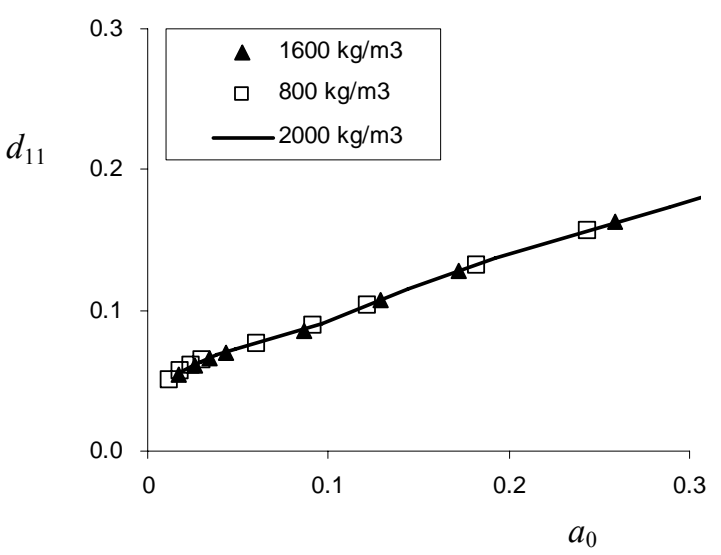

FIGURE 10

EFFECT OF SOIL DENSITY

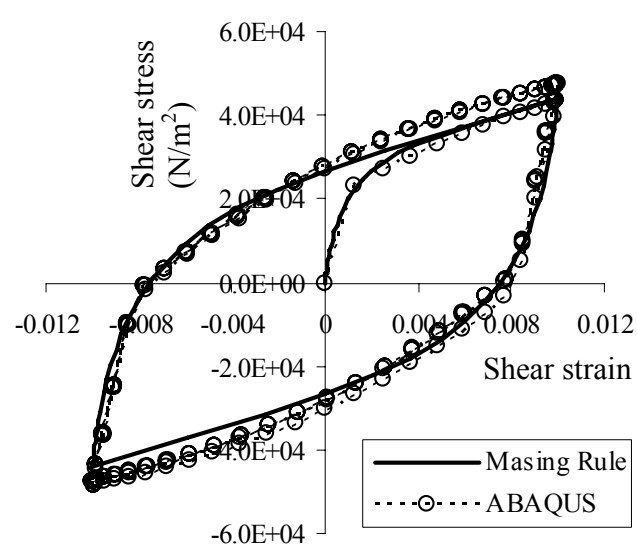

FIGURE 9

ABAQUS SIMULATION OF SIMPLE SHEAR

Besides the ratio $U_{0} / b$, soil properties also affect the nonlinear behavior of strip foundation. Figure 12 shows the static responses of a strip foundation of $b=2 \mathrm{~m}$ under vertical harmonic displacement of amplitude $0.04 \mathrm{~m}$ with soil material Cases A, B, C and D respectively.

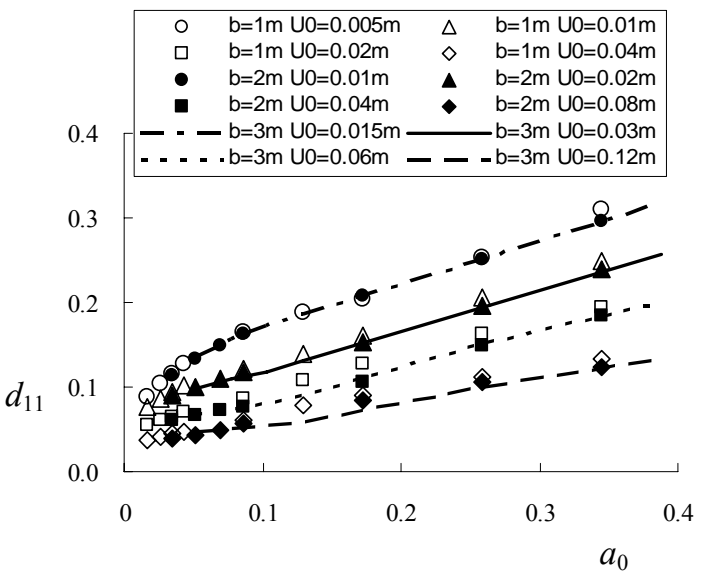

FIGURE 11

EFFECT OF RATIO $U_{0} / b$ 
Dynamic analyses were performed on a strip foundation with half-width of $2 \mathrm{~m}$ sitting on different soil and subjected to different $U_{0} / b$ ratios. Its dynamic stiffness parameter, $d_{11}$, is plotted in Figure 13. It is observed that none of the initial stiffness, yield stress and post-yielding stiffness dominates individually the global trend of the nonlinear radiation curve family. Instead of the material-level nonlinear properties, the extent of the nonlinearity developed in the foundation-soil system determines how far the radiation damping of the nonlinear soil differs from that of linear soil.

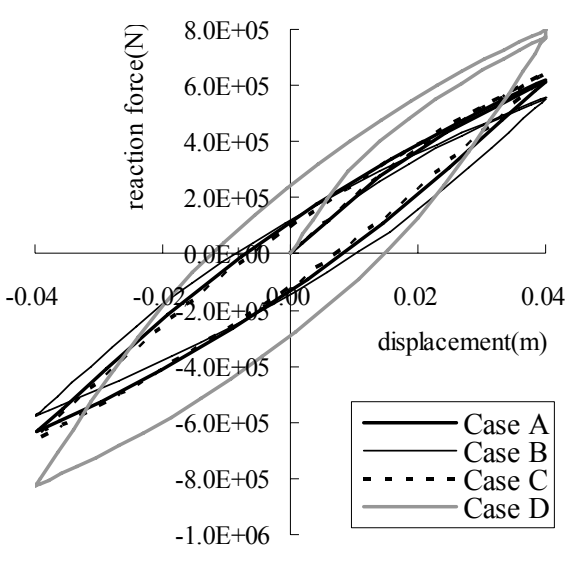

FIGURE 12

STATIC CYCLIC BEHAVIOR OF FOUNDATION

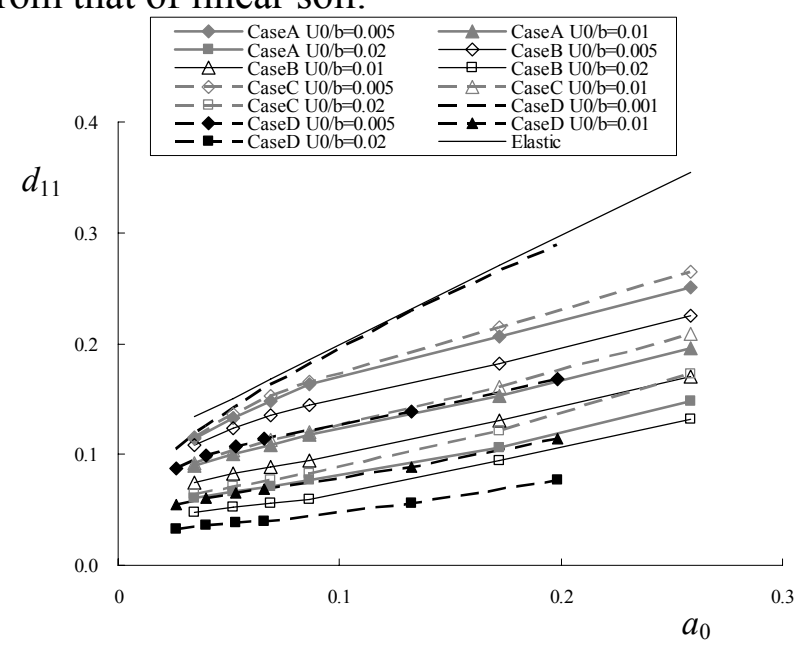

FIGURE 13

Hysteretic damping ratio (as defined in (10)) is a possible choice to represent the development of material nonlinearity. The corresponding hysteretic damping ratios $\xi$ for various combinations of soil cases and $U_{0} / b$ ratios used in Figure 13 are shown in Table 1. Larger hysteretic damping ratio indicates more nonlinearity in soil. It can be concluded that the radiation damping decreases monotonically with the increase of hysteretic damping ratio, i.e. soil nonlinearity. Essentially, the hysteretic damping ratio represents the combined effects of $U_{0} / b$ and nonlinear soil properties at the global level.

\begin{tabular}{ccccc}
\hline & $U_{0} / b=0.001$ & $U_{0} / b=0.005$ & $U_{0} / b=0.01$ & $U_{0} / b=0.02$ \\
\hline Soil Case A & - & $0.32 \%$ & $3.71 \%$ & $8.14 \%$ \\
Soil Case B & - & $1.30 \%$ & $6.09 \%$ & $10.14 \%$ \\
Soil Case C & - & $0.29 \%$ & $3.25 \%$ & $6.73 \%$ \\
Soil Case D & $0.01 \%$ & $3.56 \%$ & $9.60 \%$ & $15.18 \%$ \\
\hline
\end{tabular}

TABLE 1

HYSTERETIC DAMPING RATIO FOR DIFFERENT LOADING COMBINATIONS

Based on the relationship between nonlinear radiation damping curves shown in Figure 13 and the corresponding hysteretic damping ratios in Table 1, a simple formula is suggested below to approximate dimensionless nonlinear radiation damping under vertical vibration:

$$
d_{11}=\lambda_{0} e^{-4.9977 \xi-0.5365} \cdot a_{0}+\mu_{0} e^{-10.3144 \xi+0.0769}, \quad 0.3 \% \leq \xi \leq 15 \%
$$

where $\lambda_{0}$ and $\mu_{0}$ is the slope and intersection of $d_{11}-a_{0}$ curve of elastic soil and can be obtained by (7). Equation (11) is used to predict radiation damping of a strip foundation 
sitting on a different soil material (La Cienega site) whose soil modulus reduction curve has been obtained experimentally [23]. Figure 14 shows the good agreement of simple shear behavior as predicted by ABAQUS and the experimental data. Figure 15 plots the predicted radiation damping using (11) and shows excellent agreement with the finite element results.

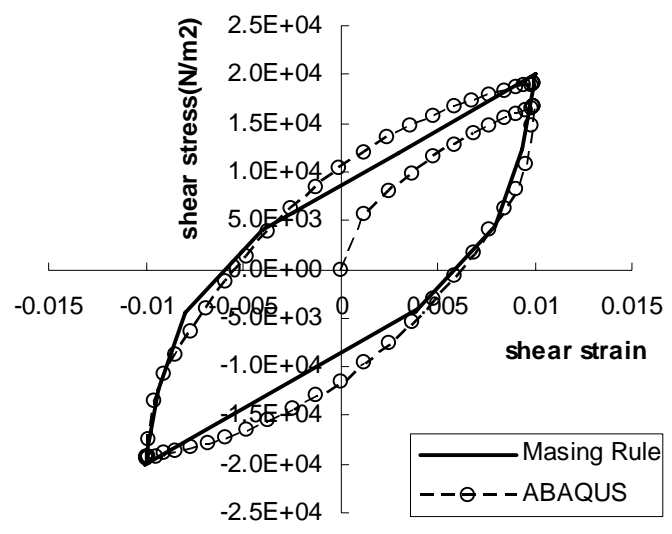

FIGURE 14

SiMPLE SHEAR OF SOIL SAMPLE AT LA CIENEGA SITE

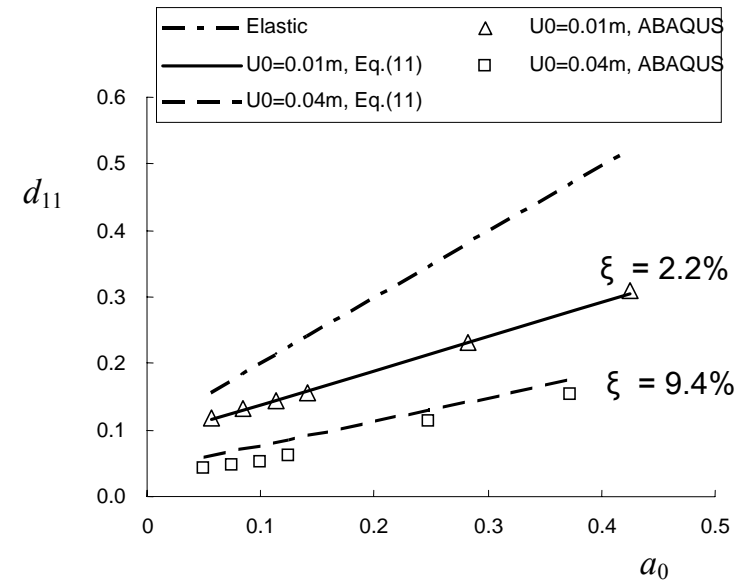

FIGURE 15

PREDICTED AND COMPUTED NONLINEAR RADIATION DAMPING OF STRIP FOUNDATION ON LA CIENEGA SOIL

\section{CONCLUSIONS}

In this study, the dynamic stiffness of strip foundation on linear and nonlinear soil medium is analyzed by finite element method. The numerical results from FEM are compared well with the theoretical solution for elastic soil with judicious choices of appropriate domain scale, mesh size and boundary conditions. Closed-form formulas are then developed to describe the dynamic stiffness of linear soil as function of frequency, foundation width, Young's modulus and Poisson's ratio. The numerical results of nonlinear soil show that the energy dissipation depends on amplitude and frequency of input motion and soil nonlinearity. The study investigated the effects of density, initial elastic stiffness, yielding stress and post-yielding stiffness and revealed that hysteretic damping ratio can be used as a global indicator to account for the reduction of radiation damping due to nonlinearity. An approximation formula is developed and showed excellent prediction when applied to different soil material.

\section{ACKNOWLEDGEMENT}

Partial financial support for this study was provided by National Science Foundation under grant NEESR-0421577.

\section{REFERENCES}

[1] Kramer, S. (1996), Geotechnical earthquake engineering, Prentice Hall, Upper Saddle River, NJ.

[2] Luco, J.E. and Westmann R.A. (1972), "Dynamic response of a rigid footing bonded to an elastic half space", Journal of Applied Mechanics, ASME, 39(2):527-534. 
[3] Hryniewicz, Z. (1981), "Dynamic response of a rigid strip on an elastic half-space", Computer Methods in Applied and Mechanical Engineering, 25(3):355-364.

[4] Gazetas, G. and Roesset, J.M. (1979), "Vertical vibrations of machine foundations", Journal of the Geotechnical Engineering Division, ASCE, 105(GT12):1435-1454.

[5] Gazetas, G. (1981), "Strip foundations on cross-anisotropic soil layer subjected to static and dynamic loading", Geotechnique, 31(2):161-179.

[6] Luco, J.E. and Westmann R.A. (1971), "Dynamic response of circular footings", Journal of Engineering Mechanics Division, ASCE, 97(EM5):1381-1395.

[7] Veletsos, A.S. and Verbic, B. (1974), "Basic response functions for elastic foundations", Journal of Engineering Mechanics, ASCE, 100(EM2):189-202.

[8] Veletsos, A.S. and Verbic, B. (1973), "Vibration of viscoelastic foundations", Earthquake Engineering and Structural Dynamics, 2(1):87-102.

[9] Wong, H.L. and Luco, J.E. (1976), "Dynamic response of rigid foundations of arbitrary shape", Earthquake Engineering and Structural Dynamics, 4(6):579-587.

[10] Wong, H.L. and Luco, J.E. (1985), "Tables of Impedance Functions for square foundations on layered media", Soil Dynamics and Earthquake Engineering, 4(2):64-81.

[11] Pais, A. and Kausel, E. (1988), "Approximate formulas for dynamic stiffnesses of rigid foundations", Soil Dynamics and Earthquake Engineering, 7(4):213-227.

[12] Gazetas, G. (1991), "Formulas and charts for impedances of surface and embedded foundations", Journal of Geotechnical Engineering, ASCE, 117(9):1361-1381.

[13] Mylonakis, G., Nikolaou, S. and Gazetas, G. (2006), "Footings under seismic loading: analysis and design issues with emphasis on bridge foundations", Soil Dynamics and Earthquake Engineering, 26(9):824-853.

[14] Borja, R.I., Wu, W-H. and Smith, H.A. (1993), "Nonlinear response of vertically oscillating rigid foundations", Journal of Geotechnical Engineering, ASCE, 119(5):893-911.

[15] Borja, R.I. and Wu, W-H. (1994), "Vibration of foundations on incompressible soils with no elastic region", Journal of Geotechnical Engineering, ASCE, 120(9):1570-1592.

[16] Kuhlemeyer, R.L. and Lysmer, J. (1973), "Finite element method accuracy for wave propagation problems", Journal of Soil Mechanics and Foundations Division, ASCE, 99(SM5):421-427.

[17] Lysmer, J., Udaka, T., Tsai, C.F., and Seed, H.B. (1975), "FLUSH: a computer program for approximate 3-D analysis of soil-structure interaction problems", Report EERC 75-30, Earthquake Engineering Research Center, University of Calfornia, Berkeley, 83pp.

[18] Lysmer, J. and Kuhlemeyer R.L. (1969), "Finite dynamic model for infinite media", Journal of Engineering. Mechanics Division, ASCE, 95(4):859-877.

[19] Lynn, P.P. and Hadid H. A. (1981), "Infinite elements with 1/rn type decay", International Journal for Numerical Methods in Engineering, 17(3):347-355.

[20] Masing, G. (1926), "Eigenspannungen und Verfertigung beim Messing", Proceedings, $2^{\text {nd }}$ International Congress on Applied Mechanics, Zurich.

[21] Zhang, J. and Makris, N. (2002), "Kinematic response functions and dynamic stiffnesses of bridge embankments", Earthquake Engineering and Structural Dynamics, 31(11):1933-1966.

[22] Wen, Y-K. (1976), "Method for random vibration of hysteretic systems", Journal of Engineering Mechanics Division, ASCE, 102(EM2): 249-263.

[23] Vucetic, M., Hsu, C.C. and Doroudian, M. (1998). "Results of cyclic and dynamic simple shear tests on soils from La Cienega site conducted for ROSRINE project and other research purposes", Report No. UCLA/ENG-98-200, University of California, Los Angeles. 